\title{
String breaking in Lattice QCD *
}

\author{
E. Laermann ${ }^{\mathrm{a}}$ with C. DeTar ${ }^{\mathrm{b}}$, O. Kaczmarek ${ }^{\mathrm{a}}$ and F. Karsch ${ }^{\mathrm{a}}$ \\ ${ }^{\text {a} F a k u l t a ̈ t ~ f u ̈ r ~ P h y s i k, ~ U n i v e r s i t a ̈ t ~ B i e l e f e l d, ~ P . O . ~ B o x ~} 100$ 131, 33501 Bielefeld, Germany \\ ${ }^{\mathrm{b}}$ Department of Physics, University of Utah, Salt Lake City, UT 84112, USA
}

The separation of a heavy quark and antiquark pair leads to the formation of a tube of flux, or string, which should break in the presence of light quark-antiquark pairs. This expected zero temperature phenomenon has proven elusive in simulations of lattice QCD. We present simulation results that show that the string does break in the confining phase at nonzero temperature.

In the absence of light quarks the heavy quarkantiquark potential is known quite accurately from numerical simulations of lattice quantum chromodynamics [1]. At large separation $R$, the potential rises linearly, as expected in a confining theory. In the presence of light quarks it is expected that the string between the heavy quark-antiquark pair breaks at large distance. All the existing lattice data at zero temperature [2,3] agree in that they do not show any indication of string breaking which would be signalled by a tendency of the potential to level off at large distances. The distances covered so far extend up to $R \lesssim 2 \mathrm{fm}$ while it has been proposed that the dissociation threshold would be reached at separations somewhere between 1.5 and $1.8 \mathrm{fm}$ [3. 1 .

We have simulated QCD with two light flavours of staggered dynamical quarks on lattices of size $16^{3} \times 4$ (new work) and $12^{3} \times 6$ (configurations from Ref. 5) at fixed values for the quark mass of $m_{q} / T=0.15$ and 0.075 respectively. The couplings were chosen to cover temperatures $T$ below the critical temperature $T_{c}$ in the range of approximately $0.7 T_{c}<T<T_{c}$. The (temperaturedependent) heavy quark potential $V(R, T)$ was extracted from Polyakov loop correlations

$$
\left\langle L(\overrightarrow{0}) L^{\dagger}(\vec{R})\right\rangle=c \exp \{-V(|\vec{R}|, T) / T\}
$$

where $L(\vec{x})=\frac{1}{3} \operatorname{tr} \prod_{\tau=0}^{\mathrm{N}_{\tau}-1} \mathrm{U}_{0}(\overrightarrow{\mathrm{x}}, \tau)$ denotes the Polyakov loop at spatial coordinates $\vec{x}$. In the limit $R \rightarrow \infty$ the correlation function should ap-

\footnotetext{
*Poster presented at the XVI International Symposium on Lattice Field Theory, Boulder, CO, July 13-18, 1998.
}

proach the cluster value $|\langle L(0)\rangle|^{2}$ which vanishes if the potential is rising at large distances (confinement) and which acquires a small but finite value if the string breaks.

In Figures 1 and 2 our data for the potential are presented in lattice units at the values of $\beta$ analyzed. The critical couplings $\beta_{c}$ have been determined as 5.306 for $N_{\tau}=4$ and 5.415 for $N_{\tau}=6$ respectively. The Polyakov loop correlations have been computed not only for on-axis separations but also for a couple of off-axis distance vectors $\vec{R}$. Rotational invariance is reasonably well recovered if one uses the lattice Coulomb behaviour to determine the quark-antiquark separation, $|\vec{R}|=1 / G_{\text {lat }}(\vec{R})$.

The data in Figures 1 and 2 quite clearly show a flattening of the potential at lattice distances of about 3 to 4 lattice spacings, depending on $\beta$. This confirms earlier results [6] obtained on smaller lattices of size $8^{3} \times 4$. Moreover, the height of the potential at these distances is in nice agreement already with the infinite distance cluster value, shown as the right-most data point in each of the plots.

In order to obtain a rough estimate of the corresponding temperatures in units of the critical temperature we applied the following procedure: at the given $\beta$ and $m_{q} a$ values an interpolation formula [7] was utilized to estimate the vector meson mass $m_{V} a$ in lattice units as well as the ratio of pseudoscalar to vector meson mass, $m_{P S} / m_{V}$. By means of a phenomenological formula which interpolates between the (experimen- 

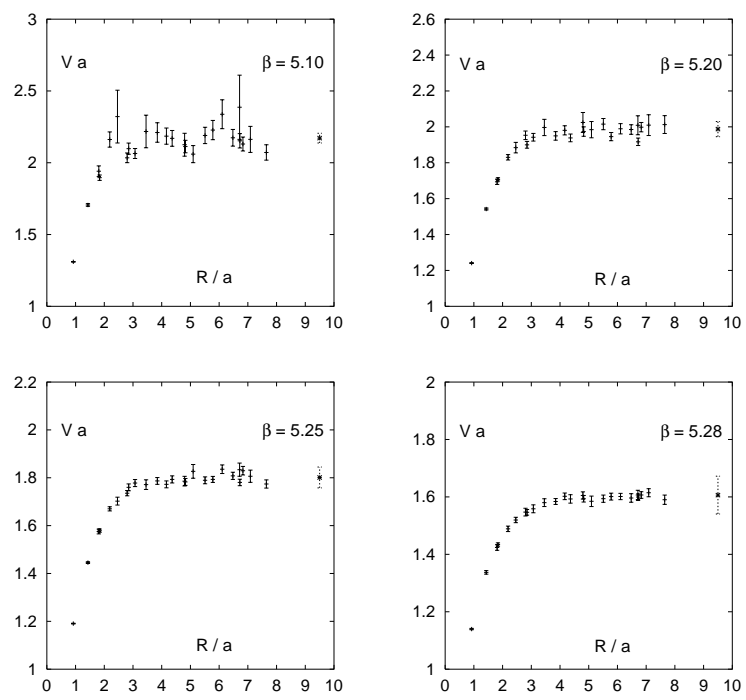

Figure 1. The potentials in lattice units at the $\beta$ values analyzed for $N_{\tau}=4$. The right-most data points plotted at $R / a=9.5$ and denoted by stars are the infinite distance cluster values $-T \ln |\langle L\rangle|^{2}$.

tally measured) $\rho$ and $K^{*}$ mass as function of the ratio $m_{P S} / m_{V}, m_{V}=756 \mathrm{MeV}+450 \mathrm{MeV} \times$ $\left(m_{P S} / m_{V}\right)^{2}$, a physical value for $m_{V}$ can be obtained. These numbers are then used to estimate the ratios of the lattice spacing at the various $\beta$.

Finally, in order to facilitate a comparison of the $N_{\tau}=4$ and 6 results with each other and with quenched data, the absolute scale was determined from a conventional Wilson loop measurement of the string tension at zero temperature at the critical $\beta_{c}$ values. The Wilson loops did not show string breaking at the separations which could be explored. The results for the critical temperature in units of the string tension are obtained as $T_{c} / \sqrt{\sigma}=0.436(8)$ for $N_{\tau}=4$ and $T_{c} / \sqrt{\sigma}=0.462(9)$ for $N_{\tau}=6$ [8].

In Figure 3 we show the potential in the presence of dynamical quarks in physical units. The potential is flat within the error bars at distances larger than about $1 \mathrm{fm}$. It also seems that the turn-over point is slightly $T$ dependent, becoming smaller with increasing temperature.

In Figure 4 quenched and full QCD potentials
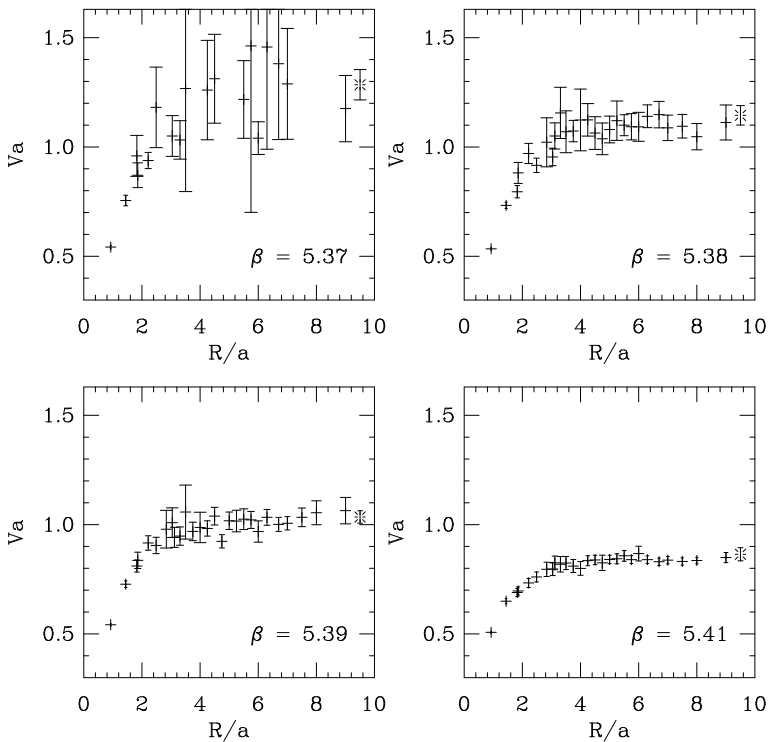

Figure 2. Same as Figure 1 except for $N_{\tau}=6$.

are compared. The quenched data has been taken from [9] and was obtained in the same way, i.e. computed from Polyakov loop correlations. Figure 4 contains, for further comparison, the dashed line denoting $-\pi /(12 R)+(420 \mathrm{MeV})^{2} R$ which gives a good description of the zero temperature quenched potential. Note that the finite temperature quenched potential is rising with distance $R$ but the slope decreases with temperature, i.e. the (quenched) string tension is temperature dependent and becomes smaller closer to the critical $T_{c}$. Again, the comparison with quenched potentials at the same temperature demonstrates that the potential in the presence of dynamical quarks becomes flat within the error bars at distances of about $1 \mathrm{fm}$. From Figure 1 we conclude that the observed string breaking, albeit at finite temperature, is an effect caused by the presence of dynamical fermions.

We have seen that string breaking is relatively easy to observe in the Polyakov loop correlation, while it is difficult to detect through the conventional Wilson loop observable. Why is this so? The Wilson loop observable creates a static quark-antiquark pair together with a flux tube joining them. In the presence of such a static pair 


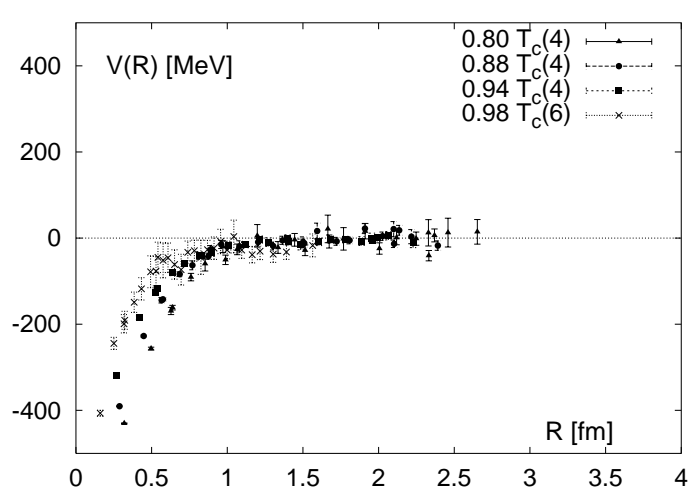

Figure 3. The potential in physical units at various temperatures. The results are from lattices with $N_{\tau}=4$ and 6 , indicated by the number in brackets. The data has been normalized to the cluster value.

at large $R$, we expect the correct ground state of the Hamiltonian to consist of two isolated heavylight mesons, however. Such a state with an extra light dynamical quark pair has poor overlap with the flux-tube state, so it is presumably revealed only after evolution to a very large temporal separation. An improved Wilson-loop-style determination of the heavy quark potential in full QCD would employ a variational superposition of the flux-tube and two-heavy-meson states 10,11. The Polyakov loop approach, on the other hand, although limited in practical application to temperatures close to or above $T_{c}$, builds in no prejudices about the structure of the static-pair ground state wave function. Screening from light quarks in the thermal ensemble occurs readily.

\section{REFERENCES}

1. G. Bali and K. Schilling, Phys. Rev. D46 (1992) 2636, D47 (1993) 661; S.P. Booth et al. (UKQCD Coll.), Phys. Lett. B294 (1992) 385; Y. Iwasaki et al., Phys. Rev. D56 (1997) 151; B. Beinlich et al., hep-lat/9707023; R.G. Edwards, U.M. Heller and T.R. Klassen, Nucl. Phys. B517 (1998) 377

2. K.D. Born et al., Phys. Lett. B329 (1994) 325; U.M. Heller et al., Phys. Lett. B335 (1994)

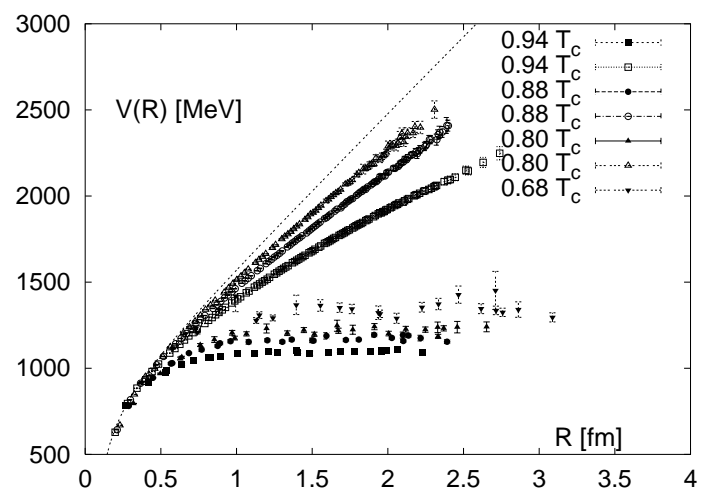

Figure 4. The potential in physical units at various temperatures. Compared are quenched (open symbols) and full (filled symbols) QCD potentials at the same temperature. The dashed line is the zero temperature quenched potential. The data has slightly been shifted as to agree at distances around $0.3 \mathrm{fm}$.

71; U. Glässner et al. (SESAM Coll.), Phys. Lett. B383 (1996) 98; C. Bernard et al. (MILC Coll.), Phys. Rev. D56 (1997) 5584; S. Aoki et al. (CP-PACS Coll.), Nucl. Phys. B(Proc. Suppl.)63A-C (1998) 221.

3. M. Talevi et al. (UKQCD Coll.), Nucl. Phys. B(Proc. Suppl.)63A-C (1998) 227.

4. C.Alexandrou et al., Nucl.Phys.B414(94)815.

5. T. Blum (for the MILC Collaboration), Nucl. Phys. B (Proc. Suppl.) 47 (1996) 503; C. Bernard et al. (MILC) Phys. Rev. D55 (1997) 6861.

6. W. Sakuler et al. Phys. Lett. B276 (1992) 155; W. Bürger et al., Phys. Rev. D47 (1993) 3034.

7. C. Bernard et al. (MILC Coll.), Phys. Rev. D54 (1996) 4585.

8. C. Bernard et al. (MILC Coll.), Phys. Rev. D56 (1997) 5584.

9. O.Kaczmarek, Diploma thesis, Bielefeld 1998. 10. I. Drummond, hep-lat 9805012 .

11. O. Philipsen and H. Wittig, hep-lat 9807020; F. Knechtli and R. Sommer, hep-lat 9807022 . 